\title{
A Micromechanics Model for Material Property of Composite
} Li-Chun BIAN $^{1, a,{ }^{*}}$, Jing PAN ${ }^{1}$, De-Liang MENG ${ }^{1}$

${ }^{1}$ Key Laboratory of Mechanical Reliability for Heavy Equipments and Large Structures of Hebei Province, Yanshan University, Qinhuangdao 066004, PR China

abianlic@126.com

${ }^{*}$ Corresponding author

Keywords: Particle, Composite, Debonding, Interphase, Elastic modulus.

\begin{abstract}
In this paper, a constitutive model of particulate-reinforced composites (PRCs) has been developed, which can be used to describe the evolution of interfacial debonding damage between particles and matrix. The influences of particle size and interphase on the equivalent elastic modulus of PRCs are analyzed and discussed. This study shows that the effective elastic modulus of composite is depended on the particle size due to the interphase.
\end{abstract}

\section{Introduction}

To-date, many researchers have attempted to estimate the effective elastic modulus of composites using both analytical and numerical methods ${ }^{[1-3]}$. The dimensions and volume fractions of interphase between particles and matrix are comparable with those of reinforcements and matrix. It is found that the interphase affects the mechanical behaviors of composites in engineering applications. The size effects of reinforcements on overall deformation and mechanical behavior of composites were also discussed by researchers. Togho et al. ${ }^{[4]}$ established an incremental damage model, which is based on Mori-Tanaka's mean field concept. Moreover, it can describe the evolution of debonding damage and the effect of particle size on the damage of composites is also discussed.

In the present investigation, for the effective elastic modulus of PRCs, the analysis method proposed by Bian and Wang ${ }^{[5]}$ has been extended to three-phase composites by introducing an interphase between particles and matrix. Moreover, the modified model, which is based on Eshelby's equivalent inclusion method and self-consistent method, can describe the influence of interfacial debonding damage between particles and matrix. As a result, the effective elastic modulus of PRCs is predicted, and the influences of particle size and interphase properties on the equivalent elastic modulus of PRCs are also discussed.

\section{Three-phase Damage Model}

It is an event that three phases of particle, matrix and interphase between them are involved in a composite. The interphase volume fraction $f_{\mathrm{I}}$ is related to that of particles $f_{\mathrm{P}}$ by

$$
\frac{f_{\mathrm{I}}}{f_{\mathrm{P}}}=\left(\frac{\mathrm{d}_{\mathrm{P}}+2 t}{\mathrm{~d}_{\mathrm{P}}}\right)^{3}-1
$$

here $d_{\mathrm{p}}$ and $t$ are particle diameter and interphase thickness, respectively. In the present analysis, it is assumed that the volume fraction of interphase is related to the surrounded particles. Then, the elastic incremental stress-strain relations of an isotropic matrix, interphase and particles can be defined as follows:

$$
d \sigma^{i}=\mathrm{L}_{i}\left(E_{i}, v_{i}\right) d \varepsilon^{i} . \mathrm{i}=\mathrm{M}, \mathrm{I} \text { or } \mathrm{P}
$$

where $d \sigma^{i}$ and $d \varepsilon^{i}$ are the incremental stress and strain, respectively, and $\mathrm{L}_{i}\left(E_{i}, v_{i}\right)$ is the 
stiffness tensor. The above equations replaced $\mathrm{i}$ with $\mathrm{M}$, I and $\mathrm{P}$ stand for the isotropic matrix, interphase and particles. $E_{i}$ and $v_{i}$ are Young's moduli and Poisson's ratios of the constituents, respectively. For the stress and strain of composite, the particle, interphase and matrix are represented with superscripts "P", "I" and "M", respectively, and those of the composite are shown by symbols without superscript.
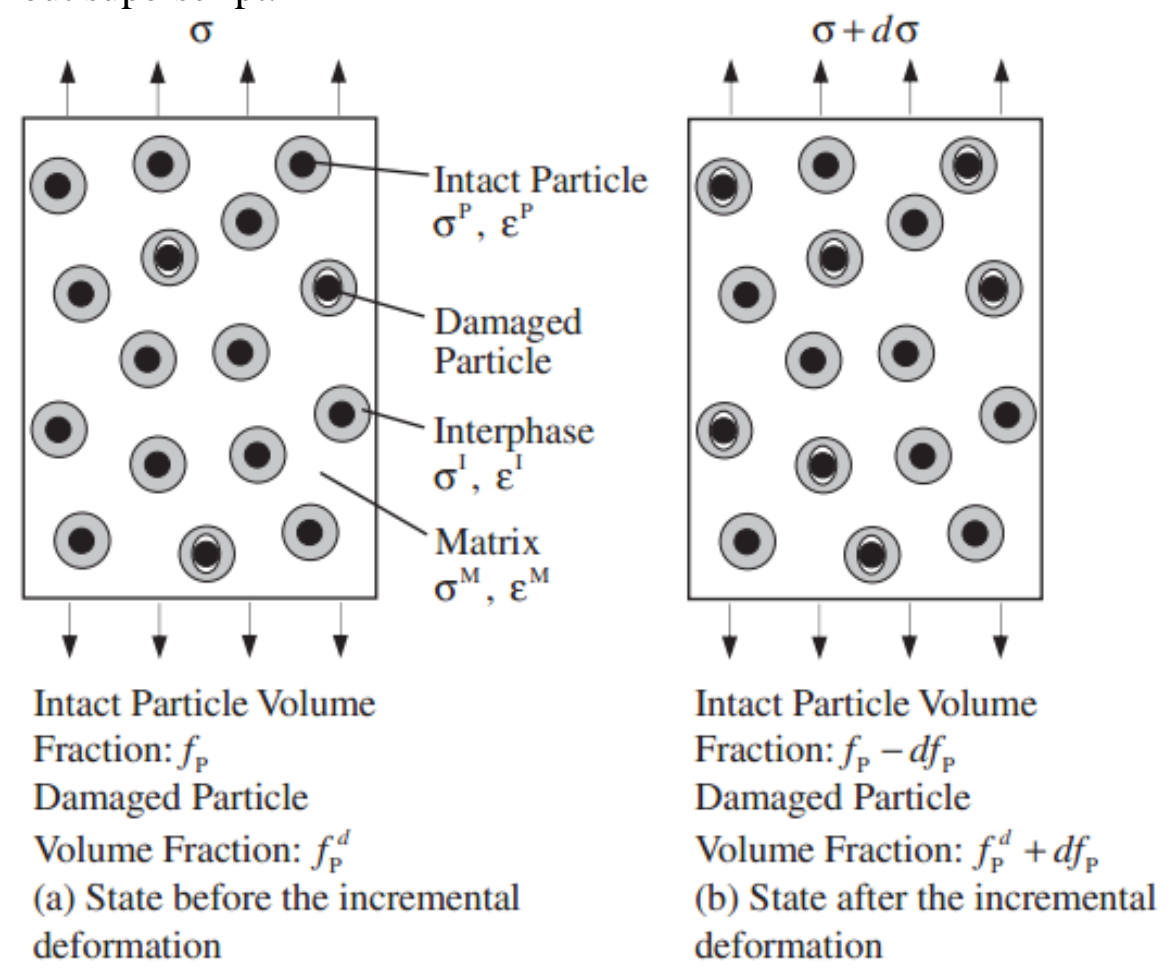

Fig.1 The States of Composite Undergoing Damage Process before and after Incremental Deformation

Fig. 1 shows the state before and after incremental deformation of PRCs in the damage process, where a constant macroscopic stress $\sigma$ and its increment $d \sigma$ are applied on the boundary of composites. The debonding damage is supposed to occur between particle and interphase in this model. The states before deformation are described in terms of the volume fractions of the intact and debonded particles $f_{\mathrm{P}}^{d}$. If the volume fraction of particle debonded during the incremental deformation is denoted by $d f_{\mathrm{P}}$, the state after deformation is described in terms of the volume fractions of intact and debonded particles $f_{\mathrm{P}}-d f_{\mathrm{P}}$ and $f_{\mathrm{P}}^{d}+d f_{\mathrm{P}}$, respactively. The composite undergoes a damage process that contains the intact and debonded particle, and particles to be debonded during an incremental deformation in the matrix. As shown in Fig. 1, the parameter $d f_{\mathrm{P}}$ is a volume fraction of reinforcements damaged in the incremental deformation. It is assumed that the initial volume fraction of interphase $f_{\mathrm{I} 0}$ is $f_{\mathrm{PO}}\left[\left(1+2 t / d_{\mathrm{P}}\right)^{3}-1\right]$. In this study, $d_{\mathrm{P}}$ is particle diameter, $t$ is interphase thickness, and $f_{\mathrm{P} 0}$ is the initial volume fraction of particles, respectively.

In the following analysis, a model of an infinite matrix with one single particle and associated interphase in it has been proposed. The elastic modulus and strain of three phases are $E_{i}$ and $\varepsilon^{i}$ ( $\mathrm{i}=\mathrm{M}, \mathrm{I}, \mathrm{P})$. According to self-consistent method, the site of particle is displaced instead of an equivalent strain $\bar{\varepsilon}$, and its elastic modulus is $E_{\mathrm{M}}$, which is the same as that of matrix. Bian and Wang [5] assumed that the matrix is in a same situation as the particles, that is, the matrix and particles are both inclusions of composite. For the study of three-phase composite, it is supposed that the interphase around a particle is also the inclusion of PRC. Moreover, according to the 
analysis above, the elastic modulus of an equivalent matrix is $E_{a}$. It is the same as an average value of elastic modulus of matrix, particles, and interphase, and can be written as $E_{a}=\left(3 K_{a}, 2 G_{a}\right)$. Here, the equivalent strain is $\bar{\varepsilon}^{a}$ due to this equivalent matrix, and the equations can be defined:

$$
\begin{aligned}
& \overline{L_{P}} \cdot \varepsilon_{m n}^{P}=\left(1-f_{P}-f_{P}^{d}-f_{I}-f_{I}^{d}\right) \cdot L_{M} \cdot \varepsilon_{m n}^{M}+f_{P} \cdot L_{P} \cdot \varepsilon_{m n}^{P}+f_{I} \cdot L_{I} \cdot \varepsilon_{m n}^{I}+f_{I}^{d} \cdot L_{I} \cdot \varepsilon_{m n}^{I-d} \\
& \varepsilon_{m n}^{i}=A_{i} \cdot \overline{\varepsilon_{m n}}, \mathrm{i}=\mathrm{M}, \mathrm{I}, \mathrm{P}
\end{aligned}
$$

where $\varepsilon_{m n}^{i}$ is the strain of i-phase inclusion, which denotes the strain of matrix, particle or interphase. $\overline{\varepsilon_{m n}}$ is the average strain of a equivalent material, of which the modulus is $E_{a} . A_{i}$ is Eshelby tensor of inclusion, and is given by ${ }^{[6]}$ :

$$
A_{i}=\left(\frac{K}{K_{i}} \rho_{1}, \frac{G}{G_{i}} \rho_{2}\right)
$$

where $K$ and $G$ are the average bulk and shear modulus of composite, respectively, and are given as follows:

$$
\begin{aligned}
& K=f_{P 0} \cdot K_{P}+f_{I 0} \cdot K_{I}+\left(1-f_{P 0}-f_{I 0}\right) \cdot K_{M} \\
& G=f_{P 0} \cdot G_{P}+f_{I 0} \cdot G_{I}+\left(1-f_{P 0}-f_{I 0}\right) \cdot G_{M}
\end{aligned}
$$

where $f_{P 0}\left(=f_{P}+f_{P}^{d}\right)$ and $f_{I 0}\left(=f_{I}+f_{I}^{d}\right)$ are initial volume fractions of particle and interphase, respectively, and are defined as follows:

$$
\begin{aligned}
& \rho_{1}=\frac{K_{i}}{K(1-\alpha)+\alpha K_{i}}, \quad \rho_{2}=\frac{G_{i}}{G(1-\beta)+\beta G_{i}} \\
& \alpha=\frac{3 K}{3 K+4 G}, \quad \beta=\frac{6(K+2 G)}{5(3 K+4 G)}
\end{aligned}
$$

Based on equations above and derivation procedure [5], the equivalent bulk modulus and shear modulus can be obtained as follows:

$$
\begin{gathered}
\overline{K_{t}}=\frac{\frac{\left(1-f_{P 0}-f_{I 0}\right) \cdot K_{M}}{\left(K_{M}-K\right) \alpha+K}+\frac{f_{P} \cdot K_{P}}{\left(K_{P}-K\right) \alpha+K}+\frac{f_{I 0} \cdot K_{I}}{\left(K_{I}-K\right) \alpha+K}}{\frac{1-f_{P 0}-f_{I 0}}{\left(K_{M}-K\right) \alpha+K}+\frac{f_{P}}{\left(K_{P}-K\right) \alpha+K}+\frac{f_{I 0}}{\left(K_{I}-K\right) \alpha+K}+\frac{f_{v}}{K(1-\alpha)}} \\
\overline{G_{t}}=\frac{\frac{\left(1-f_{P 0}-f_{I 0}\right) \cdot G_{M}}{\left(G_{M}-G\right) \beta+G}+\frac{f_{P} \cdot G_{P}}{\left(G_{P}-G\right) \beta+G}+\frac{f_{I 0} \cdot G_{I}}{\left(G_{I}-G\right) \beta+G}}{\frac{f_{P}}{\left(f_{P 0}-f_{I 0}\right.}+\frac{f_{I 0}}{\left(G_{M}-G\right) \beta+G}+\frac{f_{v}}{G(1-\beta)}}
\end{gathered}
$$

where the parameters for the following predictions can be found in study [5]. 


\section{Results and discussion}

In this study, the SiC particle reinforced aluminum (Al) alloy composite (SiC/A356-T4) under uniaxial tension is employed for the theoretical analysis. Based on the model developed, the effective elastic modulus of composites is predicted. In the present investigation, the material properties of $\mathrm{SiC} / \mathrm{Al}$ composite are presented in Table 1.

Tab.1 The Material Properties of SiC/Al Composite

\begin{tabular}{cccc}
\hline Parameter & Al(matrix $)$ & SiC(particle) & Interphase \\
\hline Young's modulus $(\mathrm{GPa})$ & 70 & 427 & 250
\end{tabular}

Poisson's ratio

0.33

0.17

0.33

The particle size effects on the effective elastic modulus without debonding damage are shown in Fig.2. The present predictions are also compared with the results of Jiang et al [3]. The interphase thickness is assumed to be $2.5 \mu \mathrm{m}$ in the numerical computation. The particle sizes are $10 \mu \mathrm{m}, 20 \mu \mathrm{m}$, $30 \mu \mathrm{m}, 40 \mu \mathrm{m}, 50 \mu \mathrm{m}, 60 \mu \mathrm{m}$ and $70 \mu \mathrm{m}$, respectively. The particle size effect on effective elastic modulus can be found, as shown in Fig. 2. It is interesting to note that if the particle size is smaller, the effective elastic modulus is higher. Moreover, the present effective elastic modulus calculated with volume fractions has a good agreement with the results from the work [3] but is somewhat higher in some cases.

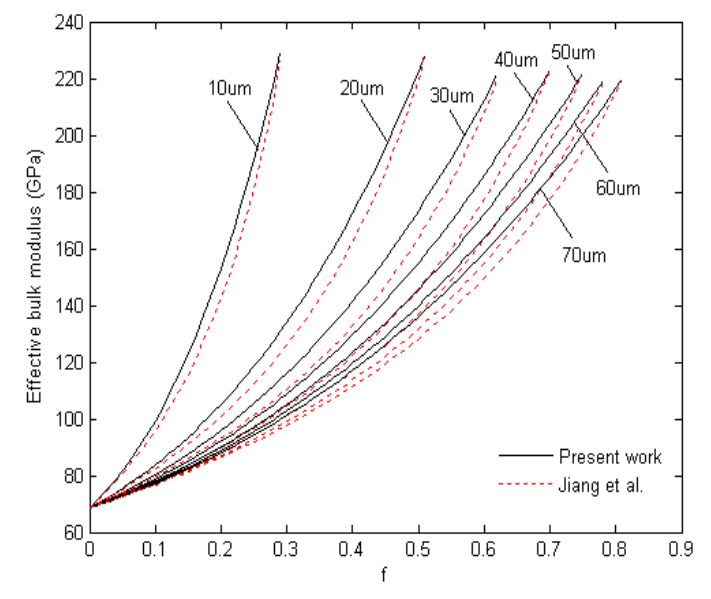

(a) Effective Bulk Modulus $\overline{K_{t}}$

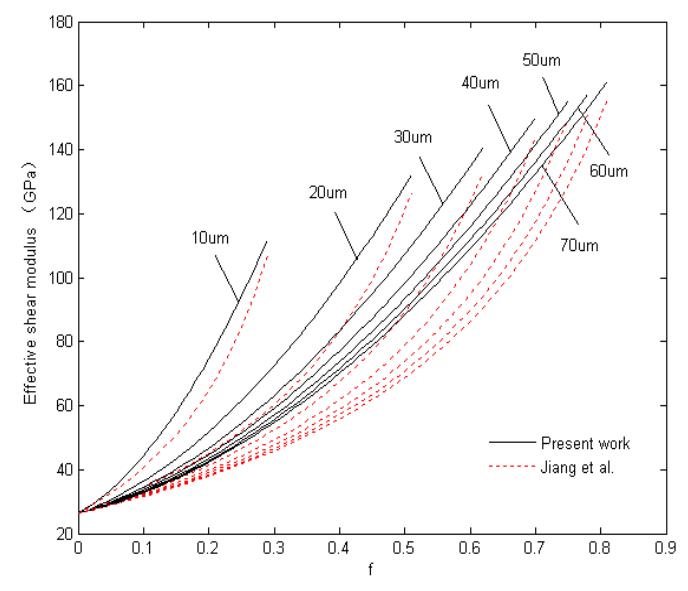

(b) Effective Shear Modulus $\overline{G_{t}}$

Fig.2 Effective Elastic Modulus with Different Particle Sizes

\section{Conclusions}

The interphase between particle and matrix is incorporated with the damage model as a third phase, and the effective elastic moduli of three-phase composites are obtained. The effective elastic modulus calculated in the present work has, in most cases, a good agreement with the existing data. Furthermore, the present investigation shows that the effective elastic modulus of composite is depended on the particle size due to the interphase. 


\section{Acknowledgment}

This research was financially supported by the Hebei Natural Science Foundation of China (A2014203139).

\section{References}

[1] T.M. Koh, S. Feih, A.P. Mouritz, Experimental determination of the structural properties and strengthening mechanisms of z-pinned composite T-joints, Composite Structures. 93(9) (2011) 2222-2230.

[2] T. Morimoto, H. Iizuka, Tensile behaviors of pre-twisted composite strands, Archive of Applied Mechanics. 83(2) (2013) 193-205.

[3] Y.P. Jiang, H. Yang, K. Tohgo, Three-phase incremental damage theory of particulate-reinforced composites with a brittle interphase, Comps Struct. 93 (2011) 1136-1142.

[4] K. Togho, Y. Itoh, Y.A. Shimamura, A constitutive model of particulate-reinforced composites taking account of particle size effects and damage evolution, Vol. 41, Elsevier Ltd., 2009, 313.

[5] L. Bian, Q. Wang, Influence of the particle size and volume fraction on micro-damage of the composites, Archive of Applied Mechanics. 83(3) (2013) 445-454.

[6] K.G. Huang, Constitutive relation of solids, Tsinghua Press, 1999. 